\title{
QUANTIFICAÇÃO DE SULFADIMETOXINA EM LEITE POR CROMATOGRAFIA LÍQUIDA DE ALTA EFICIÊNCIA
}

\author{
Carlos Willian Feltrin, Ana Maria de Souza Mello, João Gabriel Rosa dos Santos, Marcelo Volpatto Marques, Nair \\ Maria Seibel e Luiz Antonio Mazzini Fontoura* \\ Departamento de Química, Fundação de Ciência e Tecnologia, Av. das Indústrias, 2270, 94930-230 Cachoeirinha - RS, Brasil
}

Recebido em 2/2//06; aceito em 25/4/06; publicado na web em 30/8/06

\begin{abstract}
SULFADIMETHOXYNE QUANTIFICATION IN MILK BY HIGH PERFORMANCE LIQUID CHROMATOGRAPHY. A method for HPLC determination of sulfadimethoxyne in milk is presented. The analyte isolation and concentration were performed by solidphase extraction through a C-8 cartridge, pre-conditioned with hexane, methanol and water and eluted with $\mathrm{MeOH}$. The recovery determination was done with a spiked solution of $20,50 \mathrm{or} 100 \mu \mathrm{g} \mathrm{L}^{-1}$. In this concentration range, the recovery was $83.2 \%$ with a RDS of $15.4 \%$. For quantification, a Zorbax Eclipse XDB-C8 (4.6 mm x $150 \mathrm{~mm}, 5 \mu \mathrm{m})$, a mobile phase of MeCN: $0.01 \mathrm{~mol} \mathrm{~L}{ }^{-1}$ $\mathrm{KH}_{2} \mathrm{PO}_{4}$ aq. (1:4), and a variable wavelength detector $(275 \mathrm{~nm})$ were used.
\end{abstract}

Keywords: sulfadimethoxyne; milk; HPLC.

\section{INTRODUÇÃO}

Sulfonamidas, assim como outras classes de antibióticos, têm sido administradas ao gado leiteiro com objetivos terapêutico, profilático ou, ainda, como estimulante de crescimento. Uma conseqüência dessa prática é a ocorrência de resíduos desses medicamentos no leite, que, mesmo em quantidades traços, podem causar problemas de saúde, como o aparecimento de reações tóxicas no consumidor ou um aumento da resistência de determinadas bactérias ao agente antimicrobiano. Segundo a Agência Nacional de Vigilância Sanitária (ANVISA), são alvo de controle sulfadimetoxina (1) sulfatiazol (2) e sulfametazina (3), tendo sido estabelecido um limite máximo de resíduo (LMR) de $100 \mu \mathrm{g} \mathrm{kg}^{-1}$ para o somatório das três sulfonamidas ${ }^{1}$. A Figura 1 apresenta as estruturas químicas das três sulfas.
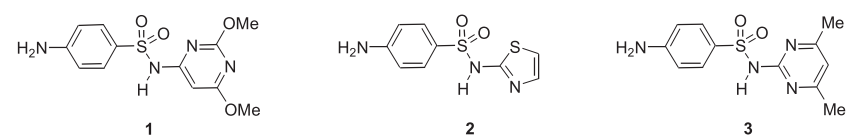

Figura 1. Estruturas da sulfadimetoxina (1), sulfatiazol (2) e sulfametazina (3)

A Associação dos Químicos Analíticos Oficiais (AOAC) descreve um método para quantificação de sulfas em leite ${ }^{2}$ que inclui os compostos 1-3. As sulfas são isoladas por extração líquido-líquido com clorofórmio/acetona (2:1) e analisadas por cromatografia líquida de alta eficiência (CLAE) em coluna C-18, tendo misturas de $\mathrm{MeOH}$ e $\mathrm{KH}_{2} \mathrm{PO}_{4}$ aq. $0,1 \mathrm{~mol} \mathrm{~L}^{-1}$ como eluente. O método, entretanto, não possibilita a quantificação simultânea das três sulfas e oferece baixa recuperação para sulfadimetoxina (1) e sulfatiazol (2). Além disso, apresenta os inconvenientes comuns à extração líquido-líquido, como formação de emulsões e grande consumo de solventes.

Alternativamente, a extração em fase sólida (EFS) tem sido bastante empregada em determinações semelhantes. Entre outras vantagens, a EFS pode levar a uma diminuição de tempo de preparo de amostra, bem como do volume total de solvente consumido no procedimento. Pode, ainda, proporcionar uma concentração do analito de interesse e a eliminação de interferentes ${ }^{3,4}$. De fato, há

*e-mail:1mazzini@uol.com.br alguns relatos na literatura sobre o uso da EFS no isolamento de sulfas em leite ${ }^{5-8}$, embora o método tenha sido, algumas vezes, criticado por sua eventual falta de reprodutibilidade ou baixa capacidade de recuperação ${ }^{7,8}$. Além da EFS em cartuchos, também a dispersão da matriz na fase sólida tem sido relatada ${ }^{7-10}$. Como alternativa à fase sólida C-189,10, outras fases como os trocadores de íons vêm encontrando emprego $0^{5,6,11}$.

Com relação ao sistema cromatográfico, colunas com fase estacionária C-18 são largamente utilizadas na análise de sulfass, ${ }^{2,-15}$. Como fase móvel, misturas de metanol ou acetonitrila com soluções aquosas de $\mathrm{KH}_{2} \mathrm{PO}_{4}, \mathrm{NaOAc}, \mathrm{NH}_{4} \mathrm{OAc}$ e HOAc, são as mais comuns ${ }^{2,6,8-12}$.

Neste trabalho, estudamos o uso de cartuchos comerciais C-8 na extração das sulfas 1-3 de amostras de leite integral e quantificação por CLAE em coluna analítica C-8. Nas condições de análise, a presença de interferentes impediu a quantificação de sulfatiazol (2) e sulfametazina (3). O método mostrou-se apropriado, por outro lado, para determinação de sulfadimetoxina (1). Foram avaliados os seguintes parâmetros: linearidade da curva analítica, linearidade da curva analítica da matriz fortificada, limite de detecção e quantificação do método, repetitividade, precisão intermediária, recuperação, estabilidade do padrão e seletividade.

\section{PARTE EXPERIMENTAL}

O equipamento utilizado foi um cromátografo a líquido de alta eficiência HP série 1100, com bomba quaternária e sistema degaseificador, válvula injetora manual com capacidade de $20 \mu \mathrm{L}$, detector UV/vis com leitura em $275 \mathrm{~nm}$, e coluna analítica Zorbax Eclipse XDB-C8 (4,6 mm x $150 \mathrm{~mm}, 5 \mu \mathrm{m})$. Os dados foram tratados através do programa de controle ChemStation (Rev. A.09.01 [1206], Agilent). A fase móvel utilizada foi acetonitrila $20 \%$ em $\mathrm{KH}_{2} \mathrm{PO}_{4}$ aq. $0,01 \mathrm{~mol} \mathrm{~L}^{-1}$ a $35{ }^{\circ} \mathrm{C}$ com o seguinte programa de vazão: $1,5 \mathrm{~mL} \mathrm{~min}^{-1}$ (0 $\left.\mathrm{min}\right), 2,0 \mathrm{~mL} \mathrm{~min}{ }^{-1}$ (3,5 $\left.\mathrm{min}\right), 2,0 \mathrm{~mL} \mathrm{~min}{ }^{-1}(8,5$ $\mathrm{min}$ ), 3,5 $\mathrm{mL} \mathrm{min}^{-1}$ (10 $\mathrm{min}$ ), com tempo total da análise de $13 \mathrm{~min}$.

Os seguintes reagentes foram utilizados: água deionizada (MilliQ, $18 \mathrm{M} \Omega \mathrm{cm}$ ), $\mathrm{KH}_{2} \mathrm{PO}_{4}$ (Nuclear), acetonitrila (EM Science), metanol (Nuclear) e padrão de sulfadimetoxina (Sigma). Para filtração da fase móvel, foram usadas membranas de nylon RC 55, com poros de $0,45 \mu \mathrm{m}$ e diâmetro de $47 \mathrm{~mm}$ (Schleicher \& Schuell). 
Para a filtração de soluções de padrões e amostras foram utilizadas membranas de nylon com poros de 0,45 $\mu \mathrm{m}$ (Sartorius, Minisart RC 25). As amostras de leite foram extraídas com cartuchos EFS Spe-ed C8 (Applied Separations, $500 \mathrm{mg}, 3 \mathrm{~mL}$ ). As soluções do padrão foram preparadas conforme indicação da literatura ${ }^{2}$.

Para a preparação de amostras fortificadas, volumes apropriados da solução padrão a $1 \mu \mathrm{g} \mathrm{mL}^{-1}$ foram adicionados sobre uma alíquota de $100 \mathrm{~mL}$ de leite.

As extrações do analito da matriz foram executadas em cartuchos EFS em fase reversa C-8. A fase sólida foi condicionada pela adição de porções sucessivas de $6 \mathrm{~mL}$ de hexano, $6 \mathrm{~mL}$ de metanol e $10 \mathrm{~mL}$ de água. A seguir, um volume de $2 \mathrm{~mL}$ de amostra (leite ou leite fortificado) foi percolado pela fase de extração. A fase sólida foi eluída com 2 $\mathrm{mL}$ de hexano, sendo o eluato desprezado. A eluição do analito retido foi realizada com $10 \mathrm{~mL}$ de metanol. O extrato alcoólico foi seco por adição de sulfato de sódio anidro e filtrado através de um funil simples para um balão-pêra. O balão contendo o extrato foi levado ao rotavapor e o solvente cuidadosamente evaporado a $32 \pm 2{ }^{\circ} \mathrm{C}$. Após a máxima evaporação, o balão foi retirado do rotavapor e purgado com um fluxo de nitrogênio para garantir a completa eliminação do solvente. A seguir, o extrato obtido foi dissolvido com $1 \mathrm{~mL}$ da fase móvel (acetonitrila $20 \%$ em $\mathrm{KH}_{2} \mathrm{PO}_{4}$ aq. $0,01 \mathrm{~mol} \mathrm{~L}^{-1}$ ). A amostra assim preparada foi filtrada através do filtro de nylon e encaminhada para análise.

Para a construção da curva analítica foram utilizados seis níveis de concentração do padrão: 50, 100, 150, 200, 300 e $500 \mu \mathrm{g}$ $\mathrm{L}^{-1}$ Cada ponto da curva representa a média de quatro medidas consecutivas. A curva analítica de amostra fortificada foi obtida a partir de amostras de leite fortificadas com o padrão com 20, 40, 60, 80 e $120 \mu \mathrm{g} \mathrm{L}^{-1}$ As amostras fortificadas foram submetidas ao procedimento de extração descrito. Também neste caso, cada ponto da curva representa a média de quatro medidas consecutivas.

$\mathrm{Na}$ determinação da recuperação, foram preparadas seis replicatas de amostras fortificadas com sulfadimetoxina nos seguintes níveis: 20, 50 e $100 \mu \mathrm{g} \mathrm{L}^{-1}$. Uma amostra de leite não fortificada foi utilizada como branco. A quantificação do analito na amostra fortificada foi realizada por padronização externa.

\section{RESULTADOS E DISCUSSÃO}

Um problema que ocorre com matrizes como o leite é o grande número de interferentes. A Figura 2 apresenta ampliações dos cromatogramas de amostras de leite branca e fortificada com sulfadimetoxina (100 $\mathrm{mg} \mathrm{mL}^{-1}$ ) submetidas ao procedimento de extração. Nas condições estudadas, a maioria dos interferentes é eluída entre 1 e 3 min. O padrão de sulfadimetoxina apresenta um tempo de retenção de 7,6 min. Não se observou a detecção de outros picos para tempos superiores a 13 min.

A faixa na qual a linearidade deve ser avaliada depende do LMR recomendado pela legislação. No caso das sulfas, o LMR é $100 \mu \mathrm{g}$

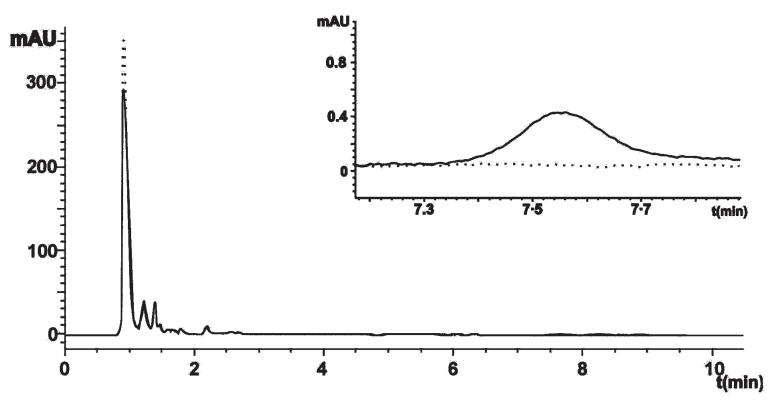

Figura 2. Cromatogramas de uma amostra branca de leite (••••) e de uma

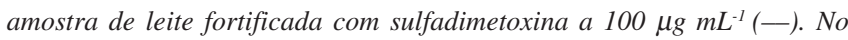
detalhe ampliado, o intervalo entre 7,2 e 7,8 min $\mathrm{kg}^{-1}$ da mistura das três. A extração em fase sólida permite aumentar a concentração do analito de interesse. No procedimento aqui proposto, a concentração obtida na amostra preparada corresponde ao dobro daquela na amostra original. Baseado nisso, a linearidade foi avaliada na faixa de 50 a $500 \mu \mathrm{g} \mathrm{L}^{-1}$. Três experimentos foram realizados, conduzidos em dias diferentes e por dois analistas distintos. Da mesma forma, três diferentes experimentos foram realizados com amostras de leite fortificadas na faixa de 20 a $120 \mu \mathrm{g} \mathrm{L}$

1. Em todos, o coeficiente de correlação encontrado foi superior a 0,99 . Valores típicos da equação da reta e dos coeficientes de correlação são apresentados na Tabela 1.

Tabela 1. Equação da reta (curvas analíticas do padrão e da matriz fortificada): coeficiente angular (a), coeficiente linear (b) e coeficiente de correlação $\left(\mathrm{R}^{2}\right)$

\begin{tabular}{lccc}
\hline & $\mathrm{a}$ & $\mathrm{b}$ & $\mathrm{R}^{2}$ \\
\hline padrão & 0,0465 & 0,2023 & 0,9999 \\
matriz fortificada & 0,0387 & 0,0379 & 1,000 \\
\hline
\end{tabular}

O limite de quantificação (LQ) representa a menor concentração da substância em exame que pode ser medida, utilizando um determinado procedimento experimental. O limite de detecção (LD), por sua vez, representa a menor concentração da substância em exame que pode ser detectada, mas não necessariamente quantificada ${ }^{16}$. Os valores de LD e LQ, 4,59 e 13,9 $\mu \mathrm{g} \mathrm{L}^{-1}$ respectivamente, foram obtidos a partir da estimativa do desvio padrão fornecida pela reta de regressão de uma curva analítica da matriz fortificada ${ }^{16}$. $\mathrm{O}$ método da $\mathrm{AOAC}^{2}$, realizado com extração líquido-líquido, é apropriado para amostras contaminadas com concentrações individuais de sulfas a partir de $5 \mu \mathrm{g} \mathrm{L} \mathrm{L}^{-1}$.

A repetitividade é o grau de concordância entre os resultados de medições sucessivas de um mesmo analito, sob as mesmas condições de medição (mesmo procedimento de medição, mesmo observador, mesmo instrumento utilizado nas mesmas condições, mesmo local e repetições em curto espaço de tempo $)^{16}$. A ANVISA, segundo Ribani et $a l .{ }^{16}$, recomenda um mínimo de nove determinações (três níveis, três repetições). Neste trabalho, a repetitividade foi determinada a partir de oito repetições em três diferentes níveis de concentração. Os resultados são apresentados na Tabela 2. A literatura recomenda valores de até $20 \%$ no caso da determinação de quantidades traço ${ }^{16}$.

As precisões intermediárias em três concentrações diferentes estimadas a partir do conjunto de cinco experimentos, realizados por dois diferentes analistas em diferentes dias, são apresentadas na Tabela 2. Novamente, em cada experimento, foram obtidas quatro medições consecutivas de cada solução de padrões nas diversas concentrações.

Tabela 2. Repetitividade e precisão intermediária na medida de padrões de sulfadimetoxina expressas através da estimativa dos desvios padrão relativos

\begin{tabular}{lcc}
\hline $\mathrm{c}\left(\mu \mathrm{g} \mathrm{L}^{-1}\right)$ & Repetitividade $(\%)$ & Precisão intermediária $(\%)$ \\
\hline 50 & 3,87 & 10,6 \\
100 & 6,72 & 6,65 \\
150 & 5,86 & 5,17 \\
\hline
\end{tabular}

A recuperação depende particularmente do método de preparação da amostra, mede a eficiência com a qual o analito é extraído de sua matriz e é usada para corrigir o valor quantificado.

A recuperação foi avaliada como a média de seis experimentos em três diferentes níveis de concentração, 20, 50 e $100 \mu \mathrm{g} \mathrm{L}^{-1}$, e valores de 76,0, 89,8 e 83,6\% foram encontrados, respectivamente. 
As estimativas do desvio padrão (s) e do desvio padrão relativo (RSD) são apresentadas na Tabela 3. No menor nível de concentração, um RSD de $24,0 \%$ foi encontrado, mas o valor cai para $6,1 \%$ em soluções fortificadas com $100 \mu \mathrm{g} \mathrm{L} \mathrm{L}^{-1}$. O método da AOAC relata a recuperação para níveis de fortificação de $20 \mu \mathrm{g} \mathrm{L}^{-1}$ ou inferiores, com uma recuperação máxima de $68,0 \%$. Para resíduos ${ }^{16}$, a recuperação é considerada aceitável na faixa de 70 a $120 \%$.

Tabela 3. Recuperação (R) de sulfadimetoxina (1) em amostras de leite fortificadas e estimativas do desvio padrão (s) e do desvio padrão relativo (RSD)

\begin{tabular}{lcrc}
\hline $\mathrm{c}\left(\mu \mathrm{g} \mathrm{L}^{-1}\right)$ & $\mathrm{R}(\%)$ & $\mathrm{s}$ & $\mathrm{RSD}(\%)$ \\
\hline 20 & 76,0 & 18,2 & 24,0 \\
50 & 89,8 & 9,3 & 10,3 \\
100 & 83,6 & 5,1 & 6,1 \\
\hline
\end{tabular}

As soluções de sulfadimetoxina nas diversas concentrações têm sido preparadas a cada três meses, aproximadamente, e curvas analíticas mistas são construídas contendo pontos do novo conjunto de padrões e também do antigo. Em todos os experimentos, o coeficiente de correlação foi sempre superior a 0,99 , indicando a adequação dos pontos à reta. Nunca foi observado, durante o período de uso das soluções, o aparecimento de outros sinais no cromatograma, além daquele atribuído ao padrão, indicando a estabilidade do mesmo.

Por fim, a seletividade foi avaliada pela determinação dos tempos de retenção de outras sulfonamidas. Nas condições de trabalho, alguns exemplos são os seguintes: sulfadiazina, 1,8 min, sulfatiazol, 1,8 min; sulfametazina, 2,7 min e sulfametoxazol, 4,3 min.

\section{CONCLUSÃO}

A utilização da extração em fase sólida (C-8) como método de isolamento e concentração de sulfadimetoxina em leite forneceu recuperações na faixa de 76 a $90 \%$. Além dos bons resultados encontrados, a técnica de extração em fase sólida apresenta como vantagens o uso de pequenos volumes de amostra e de solvente de extração, rapidez e facilidade de execução. Nas condições instrumentais descritas, a sulfadimetoxina apresenta tempo de retenção de 7,6 min, livre de interferentes.

\section{AGRADECIMENTOS}

PAMVet - ANVISA

\section{REFERÊNCIAS}

1. http//:www.anvisa.gov.br/alimentos/pamvet/pamvet.pdf, acessada em Agosto 2004.

2. Smedley, M. D.; J. AOAC. Int. 1994, 77, 1112

3. Lanças, F. M.; Extração em Fase Sólida (SPE), RiMa: São Carlos, 2004.

4. Queiroz, S. C. N.; Collins, C. H.; Jardim, I. C. S. F.; Quim. Nova 2001, 24, 68 .

5. Kennedy, D. G.; McCracken, R. J.; Cannavan, A.; Hewitt, S. A.; J. Chromatogr., A 1998, 812, 77.

6. Suhren, G.; Heeschen, W.; Anal. Chim. Acta 1993, $275,329$.

7. Furusawa, N.; J. Chromatogr., A 2000, 898, 185.

8. Takeda, N.; Akiyama, Y.; J. Chromatogr. 1992, 607, 31.

9. Le Boulaire, S.; Bauduret, J-C.; Andre, F.; J. Agric. Food Chem. 1997, 45, 2134.

10. Long, A. R.; Short, C. R.; Barker, S. A.; J. Chromatogr. 1990, 502, 87.

11. Tarbin, J. A;, Clarke, P.; Shearer, G.; J. Chromatogr., B: Anal. Technol. Biomed. Life Sci. 1999, 729, 127.

12. Rhijn, J. A. V.; Lasaroms, J. J. P.; Berendsen, B. J. A.; Brinkman, U. A. Th.; J. Chromatogr., A 2002, 960, 121.

13. Kim, D.-H.; Lee, D. W.; J. Chromatogr., A 2003, 984, 153.

14. Haller, M. Y.; Müller, S. R.; McArdell, C. S.; Alder, A. C.; Suter, M. J.-F.; J. Chromatogr., A 2002, 952, 111.

15. Grande, B. C.; Falcón, M. S. G.; Comesaña, M. R.; Gándara, J. S; J. Agric. Food Chem. 2001, 49, 3145

16. Ribani, M.; Bottoli, C. B. G.; Collins, C. H.; Jardim, I. C. S. F.; Melo, L. F. C.; Quim. Nova 2004, 27, 771. 